\title{
Performance Des entreprises informelles en Côte d'Ivoire : Quelle contribution du microcrédit?
}

\author{
Becho Isabelle \\ Doctorante en Economie, Université Félix Houphoet Boigny (UFHB), UFR des Sciences Economiques et de \\ Gestion (UFR SEG) Côte d'Ivoire
}

\begin{abstract}
Résumé: Cette étude évalue le lien entre l'accès au microcrédit et la performance des entreprises informelles en Côte d'Ivoire à partir de l'approche quasi-expérimentale basée sur la méthode d'appariement sur score de propension (PSM). Les résultats révèlent que le microcrédit a un impact positif et significatif sur la performance des entreprises informelles. L'accès au microcrédit contribue à l'augmentation du chiffre d'affaires, du résultat net et de la main d'œuvre, cependant cette contribution est faible en matière d'augmentation de la main d'œuvre. Une des raisons essentielles qui peut expliquer cette faiblesse est la politique de remboursement pratiquée par les Institutions de Microfinance (IMF). En effet, cette politique est perçue par les bénéficiaires comme une contrainte majeure dans le développement de leurs activités.

Abstract: This study assesses the link between access to microcredit and the performance of informal enterprises in Côte d'Ivoire based on the quasi-experimental approach using the PSM (Propensity Score Matching) method. The results show that microcredit has a positive and significant impact on the performance of informal enterprises. Access to microcredit contributes to increasing turnover, net income and labor force, but this contribution is small in terms of labor force growth. One of the main reasons for this weakness is the reimbursement policy practiced by Microfinance Institution (MFIs). Indeed, this policy is perceived by the beneficiaries as a major constraint in the development of their activities.
\end{abstract}

Mots clés: Approche quasi-expérimentale, Entreprises informelles, Performance, Microcrédit

\section{Introduction}

Le secteur informel peut-être défini comme toute activité qui échappe à l'État en termes d'enregistrement fiscal, il est un vecteur important des économies des pays africains et permet de suppléer les structures publiques et privées en matière de recrutement de la main-d'œuvre disponible (AFD, 2012). Il représente plus de $50 \%$ de la valeur ajoutée globale du PIB des pays à faible revenu, plus de $80 \%$ de l'emploi total et plus de $90 \%$ des emplois nouvellement créés dans ces pays (Mbaye, 2014). En Côte d'Ivoire, le secteur informel représente $91 \%$ des emplois contre seulement $5 \%$ dans le secteur privé formel et $4 \%$ dans le secteur public (ENSETE, 2013). Cependant, les activités de ce secteur ont en commun d'être vulnérables. Cette vulnérabilité est due au fait qu'elles doivent recourir du mieux possible à l'autofinancement et à des mécanismes informels de crédit pour l'accroissement de leurs activités.

La microfinance à travers sa composante microcrédit est un élément clé dans la création d'emplois pouvant augmenter les revenus de l'économie informelle, tout en étant également bénéfique pour les prestataires de ces services (OIT, 2015). Elle est peut-être considérée comme une opportunité pour lever les contraintes au développement des microentreprises, dont les risques de réussite sont confondus avec les risques d'échecs (Koloma et Ratsimalahelo, 2015). Lelart (2006) définit le microcrédit comme étant «un petit crédit, d'un montant peu élevé, sensiblement inférieur au crédit qu'une entreprise ou un ménage peut solliciter d'une banque. Ce crédit est demandé par des personnes qui disposent d'un revenu relativement bas. Il est souvent demandé pour développer une activité génératrice de revenus, qu'il s'agisse d'une ancienne activité que l'on voudrait étendre ou d'une nouvelle que l'on voudrait créer». En liant la microfinance au phénomène de la pauvreté, Barboza et Bareto (2006), affirme que le microcrédit est une alternative financière, pour les gens de la plus faible tranche de distribution du revenu, qui permet de promouvoir le développement économique en rompant le cercle de la pauvreté à travers l'accès au crédit et en stimulant l'entrepreneuriat.

Dans ce sens, Gutiérrez-Nieto et al (2005) considèrent que le microcrédit est l'offre de petits prêts pour des populations vraiment pauvres pour des projets d'auto emploi qui génèrent du revenu dans un esprit d'entrepreneuriat et d'investissement. La microfinance est aujourd'hui l'une des politiques clairement identifiées dans les Documents Stratégiques de Réduction de la Pauvreté (DSRP) pour réduire la pauvreté urbaine. (Gubert et al 2004). L'émergence d'expériences de programme de Microfinance en Côte d'Ivoire date du début des années 1990, il représente près de 39,78 \% de part de marché du secteur financier et compte 20,04 $\%$ de clients (DM, 2012). Le nombre de bénéficiaires de crédit est passé de 44411 en 2011 à 92940 en 2012, soit un taux de croissance de $109 \%$. Le montant de crédit mis en place est passé de 29,3 milliards FCFA en 2011 à 72,2 milliards de FCFA en 2012, soit une hausse $146 \%$. Au nombre des financements accordés, $64 \%$ 
sont des crédits destinés à des activités génératrices de revenus (le commerce, les services, l'artisanat...) essentiellement dans le secteur informel, $14 \%$ sont des crédits à la consommation, $12 \%$ sont des crédits à la construction et $10 \%$ sont des crédits pour l'agriculture et l'élevage (DM, 2012). Malgré la place du secteur informel dans la politique d'octroi de crédit des IMF, les activités de ce secteur sont toujours précaires près de $92,4 \%$ de ces entreprises ont un capital inférieur à 250000 FCFA. (AGEPE, 2012).

$\mathrm{Au}$ vu de ces constats, il se pose donc le problème de la contribution du microcrédit à la performance des activités financées par les IMF ? L'accès au crédit a-t-il un impact significatif sur la performance des entreprises bénéficiaires?

Cette étude a pour objectif principal d'analyser le lien entre l'accès au crédit et la performance des entreprises informelles. De façon spécifique, il s'agit d'analyser le niveau de performance des entreprises informelles bénéficiaires de crédit et non bénéficiaires, d'évaluer l'impact du crédit sur la performance de ces entreprises. L'article est organisé de la façon suivante : la section 2 fait une revue de la littérature sur le cadre théorique et empirique de l'évaluation d'impact en microfinance, la section 3 décrit la spécification du modèle et la stratégie d'estimation, la section 4 présente les données mobilisées, la section 5 discute les résultats empiriques et la section 6 présente les conclusions et fait quelques recommandations de politique.

\section{Revue de littérature}

\subsection{Cadre théorique}

Dans la littérature sur la Microfinance, deux courants de pensée s'opposent. Le premier courant est l'approche welfariste ou l'approche du bien-être et le deuxième courant est l'approche institutionnaliste (Morduch, 2000 ; Brau et Woller, 2004 ; Ejigu, 2009 ; Gutiérrez-Nieto et al, 2009). Le débat qui anime ces deux courants de pensée a pour origine les visions différentes sur ce que devrait être les rôles et les priorités des IMF pour permettre aux populations à faible revenu d'avoir accès aux services financiers dans les meilleures conditions ce que Morduch (2000) a qualifié de «microfinance schism ».

En effet l'approche welfariste mesure l'impact de la microfinance principalement par la façon dont elle répond aux besoins des plus pauvres dans le court terme ou par la réduction de la pauvreté, l'approche institutionnaliste quand a elle mesure le succès par la viabilité des institutions en supposant que les institutions de Microfinance autonomes sont susceptibles de contribuer significativement à l'expansion des revenus et à la réduction de la pauvreté.

L'approche welfariste est aussi appelée approche de «crédit dirigé » et est perçu comme faisant partie d'un programme intégré de lutte contre la pauvreté, la vulnérabilité et d'amélioration du bien-être des populations pauvres. En plus de fournir des services financiers, cette approche favorise l'octroi de services nonfinanciers comme la formation et l'assistance technique aux micro- entrepreneurs, l'alphabétisation, etc.

Les tenants de l'approche du bien-être utilisent des «welfare studies » pour évaluer l'efficacité des programmes de microcrédit. Le but recherché est de mesurer l'impact du microcrédit sur les conditions de vie des populations cibles. On veut évaluer la situation avant et après l'adhésion à l'IMF. On s'intéresse alors à l'évolution du niveau de revenus, de nutrition et d'éducation des pauvres ainsi qu'à l'accès des services de santé et d'assurance.Cependant les institutionnalistes reprochent à ce type d'études d'être trop subjectives et d'engendrer des coûts trop élevés en plus des difficultés méthodologiques qu'elles peuvent rencontrer.L'approche institutionnaliste également qualifiée d'approche de marché financier fait son apparition en réponse aux critiques émises sur l'approche welfariste. Elle inscrit les programmes de microcrédit à l'intérieur d'une logique de marché. Conscients de la capacité limitée des donateurs à répondre à la demande massive de microcrédits, les tenants de ce courant basent leurs initiatives sur deux caractéristiques essentielles: une volonté de massification du crédit ainsi qu'une volonté de pérennisation des institutions. Dans le but de développer des IMF pérennes et commerciales, ces programmes prônent l'autosuffisance et la viabilité financière des institutions. Cela se traduit par des taux d'intérêts parfois très élevés qu'on impose aux clients, justifiés par les coûts élevés de transaction relatifs à tout microcrédit. L'objectif recherché n'est pas focalisé sur l'amélioration du bien-être en général des pauvres, mais plutôt sur l'amélioration de l'accès aux services financiers de la catégorie des pauvres exclus du secteur bancaire traditionnel.En ce qui concerne l'évaluation des programmes de microcrédit, les institutionnalistes trouvent qu'il est préférable de recourir à des proxys. Par conséquent, ils procèdent plutôt à des «institutional studies ». Selon eux, la bonne santé financière et l'intérêt porté aux pauvres sont liés, l'intérêt doit essentiellement porter sur des variables de marché à savoir le nombre de pauvres touchés, le degré d'autosuffisance financière, la rentabilité, la qualité des services offerts, etc. L'accent est ainsi mis sur l'institution elle-même au détriment de sa clientèle. Les welfaristes à leur tour émettent des critiques sur la non-prise en compte de l'effet du microcrédit sur les pauvres.De ce cadre théorique, il ressort que le rôle joué par la microfinance à travers sa composante microcrédit est incontournable surtout dans un contexte d'amélioration du niveau de vie des populations en Côte d'Ivoire. Elle permet aux pauvres d'accéder à des microcrédits afin de subvenir à leur besoin quotidien et de développer leurs activités (leurs entreprises). Par conséquent notre analyse se basera sur l'approche welfariste (approche du bien-être). 


\subsection{Revue empirique}

La question de savoir si la microfinance aide réellement les pauvres est le sujet de débat le plus intense au niveau de l'industrie de la microfinance (Banerjee et al, 2009; Guérin, 2011). Cela pose alors la problématique de l'évaluation de son impact c'est-à-dire de l'étendue avec laquelle le revenu, le profit et le bien-être des populations ciblées par la microfinance augmentent (Conning, 1999). Dans la littérature portant sur la microfinance, plusieurs études affirment que l'intervention des institutions de microfinance à un impact positif sur le revenu des emprunteurs pauvres. Pour Sebstad et Chen (1996) le crédit accroît significativement les variables d'output (chiffre d'affaires, production, valeur ajoutée, profit) et l'accumulation du capital, cependant ils trouvent que ces résultats sont plus mitigés en ce qui concerne l'emploi. Le microcrédit permet d'augmenter significativement les profits et les investissements des microentreprises qui en bénéficient. De ce fait, le microcrédit semble avoir un impact sur l'emploi. En Bolivie, en l'espace de trois ans, deux tiers des clients de l'IMF CRECER ont vu leurs revenus augmenter significativement, grâce notamment à la croissance de leur activité commerciale et à leur capacité à grouper leurs achats de marchandise du fait de l'accès au crédit. (MkNelly et Dunford, 1999). Weiss et Montgomery (2005) dressent un constat globalement positif de l'impact des programmes de la microfinance sur la réduction de la pauvreté en Asie et à Amérique latine. La hausse de revenu associée à un programme du microcrédit favorise plutôt la consommation que l'investissement. En fait, cet accroissement constitue l'objet d'un impact plus douteux en termes de vulnérabilité qui figure dans des chocs d'instabilité du revenu et de la consommation. Aussi, Khandker (1998) a montré que les interventions des institutions de microfinance améliorent à la fois la consommation et la production des pauvres qui avaient des difficultés d'accès au crédit du marché formel. L'importance de l'impact du microcrédit dépend de l'utilisation qui en est faite. Il retient non seulement le financement d'une vitalité économique, mais le microcrédit concerne les crédits des micros entrepreneurs qui veulent consolider leur activité au lieu de la banque traditionnelle.

Hulme et Mosley (1996) cité par Gubert et al (2004), rassemble les études d'impact de treize IMF intervenant dans sept pays (la Bolivie, l'Indonésie, le Kenya, le Malawi, l'Inde, le Sri Lanka et le Bangladesh). Pour chacune des études, deux vagues d'enquête ont été réalisées, en 1989 et 1993, auprès d'un échantillon aléatoire de 150 emprunteurs et d'un groupe de contrôle présentant des caractéristiques similaires en termes de niveau de revenu, d'actifs et d'accès aux infrastructures. Dans l'ensemble, les études concluent à un impact positif de l'intervention des IMF sur le revenu des emprunteurs pauvres. L'augmentation moyenne du revenu des emprunteurs par rapport à celui des non emprunteurs varie entre 10-12 \% (Indonésie) et $30 \%$ (Bangladesh et Inde). Les résultats des travaux de Attanasio et al (2015) en Mongolie et de Banerjee et al (2015) en Inde à partir d'une experience randomisée montrent qu'il y a un effet positif sur les actifs et l'investissement des bénéficiaires de microcredit. De même, à partir d'une approche quasi-expérimentale les travaux de Koloma et Ratsimalahelo (2015) montrent que le chiffre d'affaires, le taux de marge, le nombre d'emplois créées sont plus important pour les microenterprises des jeunes bénéficiaires de microcredit comparativement aux nonbénéficiaires au Mali.

Cependant ces résultats ont été remis en cause par certains travaux (Angelucci et al., 2012, Augsburg et al., 2012, Crepon et al., 2011) qui trouvent que l'accès au microcrédit ne produirait que peu ou pas d'effet sur le bien-être des emprunteurs et sur la creation d'emploi. Morduch (1999) critique la manière dont ont été constitués les groupes de contrôle, il montre que, dans certaines études de cas, la situation du groupe de contrôle était dès le départ très différent de celle du groupe d'emprunteurs, avec un différentiel de revenu entre les deux groupes de $40 \%$ en moyenne au profit des emprunteurs. L'impact mesuré ne serait donc que le reflet de la persistance d'un écart de revenu entre les deux groupes, et non l'effet du crédit. Karlan (2001) dans une revue des travaux réalisés dans le cadre du projet AIMS, dont la portée est bien plus générale, relève des problèmes non correctement traités de biais de sélection de la clientèle comme des groupes témoins, d'attrition non aléatoire des enquêtes à passages répétés, de manque de contrôle de la dynamique des institutions (changements dans l'identification et le choix des clients au cours du temps, des modalités d'octroi des services fournis) ou de l'évolution conjoncturelle (croissance ou récession), toutes choses qui selon lui entachent sérieusement nombre de résultats des évaluations disponibles.

Les contradictions constatées au niveau des résultats des études d'impact qui ont déjà été menées ont entrainé beaucoup de controverses et semé la confusion dans le domaine (Roodman et Morduch, 2009), des réflexions très critiques émergent pour contester les appréciations positives faites sur la microfinance en considérant qu'elle engendre plutôt de nouvelles pressions sociales (surendettement, pression morale et suicide) (Wright-Revolledo, 2005), au lieu de contribuer à l'amélioration des conditions de vie des populations démunies. Certaines études trouvent qu'il n'y a pas de differences significatives entre les actvités du groupe traité et du groupe de contrôle. Karlan et Zinman (2011) trouvent même un effet et négatif sur les activités des bénéficiaires de microcédit. Ainsi, nombreuses sont les études qui ont documenté et observé un impact positif du microcredit, ces études d'impact montrent que toutes les innovations en microfinance ont véritablement eu des effets sur les résultats ciblés (OIT, 2015). Cependant, il en existe quelques-unes qui ont conclu à un faible impact voir même à l'absence d'impact ou même à un impact négatif, notamment dans des contextes de zones 
très pauvres focalisées sur une seule activité économique, où l'accès au crédit peut entraîner une saturation rapide du marché local.Des problèmes d'ordre méthodologique quant à l'effet de la microfinance sur les bénéficiaires ont également été relevés. Pour toutes ces raisons, l'évaluation d'impact de la microfinance a un rôle important à jouer dans le développement des activités du secteur informelles en Côte d'Ivoire et demeure un champ important pour la recherche, les décideurs politiques, les praticiens du développement, les gestionnaires des Institutions de Microfinance et les bailleurs qui veulent s'assurer que les ressources sont bien utilisées. En Côte d'Ivoire, peu d'études ont été menées en matière d'évaluation d'impact de la Microfinance. Cette étude se propose donc de combler ce déficit et de révéler les résultats accomplis par la Microfinance à travers le microcrédit dans le cadre de la promotion de l'entrepreneuriat et de la lutte contre la pauvreté

\section{Cadre Methodologique}

Cette étude a pour objectif d'évaluer l'impact du microcrédit dans l'accroissement des activités des entreprises informelles en Côte d'Ivoire. Deux méthodes de travail seront développées : La première sera fondée sur une analyse et une interprétation de l'évolution des données statistiques et surtout des indicateurs d'évolution des activités des entreprises informelles. La seconde, se fera à partir d'une modélisation économétrique fondée sur la méthode d'appariement sur score de propension et permettra d'évaluer l'impact du microcrédit sur la performance des entreprises de micro-entrepreneurs bénéficiaires en comparaison de la performance des entreprises de micro-entrepreneurs non bénéficiaires. Cette méthode a été utilisée par Roubaud et Gulbert (2004); Ghalib et al (2012); Lecoq et al (2014).

Évaluer l'impact de l'intervention d'une institution de Microfinance (IMF) revient à se poser la question suivante : en quoi la situation des entrepreneurs bénéficiaires est-elle différente de ce qu'elle aurait été dans le cas contraire? Quel est le gain que retirent leurs entreprises de cette intervention?

\subsection{Spécification du modèle}

L'objectif de cette étude est de mesurer l'impact du microcrédit sur la performance des entreprises informelles $\left(\Delta_{A T T}\right)$, en estimant la différence entre les entreprises bénéficiaires et les non-bénéficiaires. Nous appliquons l'approche standard d'appariement formalisé par Rubin (1973), défini comme suit :

$$
\Delta_{i}=Y_{i}^{1}-Y_{i}^{0}
$$

$\Delta_{i}$ est l'effet de l'accès au microcrédit de l'entreprise i, où i $=1,2, \ldots, N . Y_{i}^{1}$ et $Y_{i}^{0}$ sont les variables de résultats qui décrivent le niveau de performance d'une entreprise $i$ conditionnellement à l'accès ou non au microcrédit ( $Y^{0}$ représente le résultat contrefactuel). L'équation (1) est censée se rapprocher de la différence entre les résultats potentiels avant et après avoir obtenu du crédit pour chaque entreprise. Il est à noter que, pour chaque entreprise i dans l'équation (1), il y a un seul résultat observé et l'autre est hypothétique et non observé à partir des données. Par conséquent, l'équation (1) est modifiée pour estimer les effets moyens du microcrédit sur les entreprises bénéficiaires $\left(\Delta_{A T T}\right)$, mais aussi sur l'ensemble des entreprises $\left(\Delta_{A T E}\right)$ (un estimateur naïf). Ainsi, l'effet moyen du traitement sur l'ensemble des entreprises est :

$\Delta_{\text {ATE }}=E\left(Y_{i}^{1}-Y_{i}^{0}\right)=E\left(Y_{i}^{1} \mid T_{i}=1\right)-E\left(Y_{i}^{0} \mid T_{i}=0\right)$

L'effet moyen du traitement dans la population des entreprises bénéficiaires de microcrédit est:

$\Delta_{A T T}=E\left(Y_{i}^{1}-Y_{i}^{0} \mid T_{i}=1\right)=E\left(Y_{i}^{1} \mid T_{i}=1\right)-E\left(Y_{i}^{0} \mid T_{i}=1\right)$

On note $\mathrm{T}_{i}$ le fait de bénéficier ou non de microcrédit. Soit $T_{i}=1$ pour les entreprises bénéficiaires et $T_{i}=0$ les entreprises non-bénéficiaires.

On a $\Delta_{A T E}=\Delta_{A T T}+E\left(Y_{i}^{0} \mid T_{i}=1\right)-E\left(Y_{i}^{0} \mid T_{i}=0\right)$

Or $E\left(Y_{i}^{0} \mid T_{i}=1\right)$ et $E\left(Y_{i}^{0} \mid T_{i}=0\right)$ ne sont pas observables et donc l'identification de $\Delta_{A T T}$ nécessite l'indépendance de $Y_{i}^{0}$ et de $T_{i}$ afin que $E\left(Y_{i}^{0} \mid T_{i}=0\right)-E\left(Y_{i}^{0} \mid T_{i}=1\right)=0$

$E\left(Y_{i}^{0} \mid T_{i}=0\right)-E\left(Y_{i}^{0} \mid T_{i}=1\right)$ est un biais qui s'explique par le fait que le niveau de performance moyen des entreprises bénéficiaires de microcrédit n'aurait pas été le même que celui des entreprises non-bénéficiaires en cas d'absence de microcrédit. Autrement dit, les entreprises ayant obtenu du crédit et celles n'ayant pas obtenu du crédit ne sont pas identiques. Pour éliminer ce biais $Y_{i}^{0}$ et $T_{i}$ doivent être indépendants.

L'équation (5) définit le sous-ensemble de toutes les entreprises qui n'ont pas obtenu du crédit. Le biais est donc la différence entre l'effet du microcrédit sur les entreprises l'ayant obtenu et la différence entre l'effet du microcrédit sur les entreprises ne l'ayant pas obtenu. 


$$
\begin{aligned}
& \Delta_{A T T}-\left[E\left(Y_{i}^{0} \mid T=0\right)-E\left(Y_{i}^{0} \mid T=1\right)\right]=E\left(Y_{i}^{1} \mid T=1\right)-E\left(Y_{i}^{0} \mid T=1\right)-E\left(Y_{i}^{0} \mid T=0\right)+E\left(Y_{i}^{0} \mid T=1\right) \\
& \Delta_{A T T}-\left[E\left(Y_{i}^{0} \mid T=0\right)-E\left(Y_{i}^{0} \mid T=1\right)\right]=E\left(Y_{i}^{1} \mid T=1\right)-E\left(Y_{i}^{0} \mid T=0\right)
\end{aligned}
$$

Le biais est égal à zéro, si l'équation (7) est vérifiée

$$
E\left(Y_{i}^{1} \mid T=1\right)-E\left(Y_{i}^{0} \mid T=0\right)=0 \Leftrightarrow E\left(Y_{i}^{1} \mid T=1\right)=E\left(Y_{i}^{0} \mid T=0\right)
$$

Ainsi, pour éviter les biais dans la procédure de sélection, plusieurs méthodologies ont été introduites basées sur les hypothèses économétriques traditionnelles. Ces dernières sont fondées sur des restrictions d'hypothèse de loi distributive et de forme fonctionnelle pour identifier l'Effet Moyen de Traitement $\left(\Delta_{A T T}\right)$ et d'autres paramètres. Les estimateurs de $\Delta_{A T T}$ sont classés sous deux classes d'hypothèses pour qu'ils soient robustes (Diagne, 2006 ; Wooldridge, 2002). La première catégorie d'estimateurs est basée sur l'hypothèse d'indépendance conditionnelle et suppose «que conditionnellement aux variables observables individuelles $\mathrm{X}$, on se situe dans le cadre d'une expérience contrôlée, c'est-à-dire avec une affectation aléatoire au traitement » (Fougère, 2007) et la deuxième sur l'hypothèse du support commun.

\subsubsection{Principe et application de la méthode d'appariement sur score de propension}

Pour contrôler les problèmes d'auto-sélection, l'on choisit comme groupe de contrôle apparié au groupe des entreprises bénéficiaires de microcrédit un sous-ensemble du groupe de contrôle composé d'entreprises non-bénéficiaires dont les caractéristiques observables sont les plus appariées possible. Cette méthode fait l'hypothèse que les seules différences entre les deux groupes proviennent de leurs caractéristiques individuelles d'une part et être bénéficiaire ou non, l'effet du traitement d'autre part. Si l'on neutralise les différences selon les caractéristiques, alors il ne reste que l'effet du traitement. L'accès au traitement dans cette analyse est le fait d'être bénéficiaire de microcrédit. Comme la moyenne contrefactuelle du résultat du microcrédit sur les bénéficiaires n'est pas observée, il est important de choisir un substitut afin d'estimer l'effet moyen du traitement sur les bénéficiaires. Pour ce faire, deux hypothèses sont faites: l'hypothèse d'indépendance conditionnelle et l'hypothèse du support commun.

\subsubsection{Hypothèse d'indépendance conditionnelle}

Lorsque l'on souhaite évaluer un programme à l'aide de données d'observations (non-expérimentales), l'on fait face à deux populations, les traités et les non-traités qui diffèrent par la distribution des caractéristiques individuelles observables qui affectent vraisemblablement le traitement. L'indépendance (inconditionnelle) entre les variables latentes de résultat $\left(Y_{i}^{0}, Y_{i}^{1}\right)$ et l'affectation au traitement $\mathrm{T}$ est une hypothèse très improbable. Une condition moins restrictive consiste à considérer qu'il existe un ensemble de variables observables $\mathrm{X}$ conditionnellement auquel la propriété d'indépendance entre les résultats latents et l'affectation au traitement est vérifiée.

$\left(Y_{i}^{0}, Y_{i}^{1}\right) \perp T \mid X$

Une façon alternative de formuler cette restriction est de considérer que conditionnellement aux variables observables individuelles $X$, on se situe dans le cadre d'une expérience contrôlée, c'est-à-dire avec une affectation aléatoire au traitement. La «randomisation» serait maintenant assurée par les covariables $X$ plutôt que par un processus aléatoire d'affectation. Ainsi, la distribution de chacun des résultats potentiels est identifiable, puisque leur distribution conditionnelle aux variables observables $X$ l'est. En conséquence, il est également possible d'identifier l'ensemble des paramètres de chacune de ces distributions, et en particulier l'effet moyen du traitement, de même que l'effet moyen du traitement pour le groupe des entreprises traitées. Remarquons toutefois que, sous l'hypothèse d'indépendance conditionnelle des résultats aux variables observables $X$, la distribution des variables latentes de résultat est identifiée, mais leur loi jointe ne l'est pas. Ceci implique en particulier que les moments d'ordre supérieur à 1 de la loi de la différence $Y_{i}^{0}-Y_{i}^{1}$ (en particulier sa variance) ne sont pas identifiés.

\subsubsection{Estimation par appariement sur score de propension}

Le problème de dimension du vecteur $X$ est fortement réduit par une propriété mise en évidence par Rosenbaum et Rubin (1983). La propriété d'indépendance conditionnellement à des variables observables implique celle d'indépendance conditionnellement à un résumé de dimension, qui est la probabilité de traitement, ou score de propension à être traité, noté

$P(X)=\mathrm{P}(T=1 \mid X)$.

Cette équation est estimée en utilisant le modèle logit ou probit. Ces modèles prévoient la probabilité pour les micro-entrepreneurs d'être bénéficiaires conditionnellement à leurs caractéristiques observables. 
L'équation (8) est indiquée comme modèle de probit ou logit et exprimée comme suit :

$P(T=1 \mid X)=P\left(y^{*}>0 \mid X\right)=P(u>-X \beta \mid X)=1-G(-X \beta)=G(X \beta)$

Où $0<G(X \beta)<1$, pour toutes les valeurs des covariables $X, X \beta=\sum_{j=1}^{k} \beta j X j$ et $G$ est une fonction cumulative normale standard. L'équation (9) est non linéaire et donc l'estimateur utilisé est celui du maximum de vraisemblance. Une fois le score estimé pour toutes les entreprises de l'échantillon, l'on détermine le support commun du score de propension pour s'assurer que pour chaque entreprise bénéficiaire de crédit, il y a une entreprise non bénéficiaire et ayant le même score de propension.

L'équation (9) satisfait à l'hypothèse d'indépendance conditionnelle, dans ce cas les résultats potentiels sont indépendants du traitement en tenant compte de l'ensemble des covariables X tels que : $Y^{0}, Y^{1} \perp T \mid P(X)$, aussi bien que l'hypothèse du support commun, Ce dernier assure toutes les entreprises avec les mêmes caractéristiques dans l'échantillon ont une probabilité positive d'être bénéficiaire ou non $(0<P(T=1 \mid X)<$ 1), par conséquent, l'estimateur de PSM $\left(\Delta_{A T T}\right)$ est sans biais.

L'estimateur final pour l'effet moyen du traitement est obtenu comme la moyenne des écarts de la performance des entreprises traités et de celle de leurs contrefactuels se définit comme suit : $\Delta_{A T T}^{P S M}=E_{P(X) \mid T=1}\left[E\left(Y_{i}^{1} \mid T=1, P(X)\right)-E\left(Y_{i}^{0} \mid T=1, P(0)\right)\right]$

\subsubsection{Analyse de la sensibilité des résultats obtenus par les méthodes d'appariement}

Une fois l'appariement effectué, l'impact du microcrédit pour une entreprise est la différence de valeur de l'indicateur entre cette entreprise et son entreprise de comparaison. Pour vérifier la robustesse des résultats obtenus, le test de Rosenbaum (2002) sera utilisé pour analyser la sensibilité des estimateurs à la présence des caractéristiques inobservables. En effet, si nous supposons qu'il y a des caractéristiques non observables $U$ qui pourraient déterminer la probabilité pour une entreprise d'obtenir du microcrédit, ceci violerait l'hypothèse d'indépendance conditionnelle aux inobservables et les résultats obtenus seront quelque peu biaisés. Dans ce cas particulier, la probabilité pour une entreprise $i$ de bénéficier de microcrédit est :

$$
\pi_{i}=\pi\left(x_{i}\right)=P\left(T_{i}=1 \mid x_{i}\right)=F\left(\beta x_{i}+\gamma u_{i}\right)
$$

De ce fait, une entreprise $j$ ayant les mêmes caractéristiques observables $\left(x_{i}=x_{j}\right)$ aura un même score de propension que $i$ seulement si $\gamma=0$. On dira qu'il existe un «biais caché » dans le cas où l'entreprise $i$ et $j$ ont les caractéristiques observables identiques, mais des scores différents $\pi\left(x_{i}\right) \neq \pi\left(x_{j}\right)$. En supposant que la variable non observable $u_{i}$ soit dichotomique (Aakvik (2001)), le rapport des odds des entreprises $i$ et $j$ :

$\frac{1}{\Gamma} \leq \frac{\pi_{i}\left(1-\pi_{j}\right)}{\pi_{j}\left(1-\pi_{i}\right)} \leq \Gamma$

Où $\Gamma=e^{Y}$, si $\Gamma=1$, alors $\gamma=0$, autrement dit les entreprises ayant les mêmes caractéristiques ont les mêmes scores de propension, il n'existe pas dans ces conditions de «biais caché ». En revanche, une valeur de $\Gamma$ égale à 2 signifie que l'une des entreprises a une probabilité deux fois plus élevée par rapport à l'autre d'être bénéficiaire de microcrédit alors qu'ils ont les mêmes caractéristiques observables. Cette situation s'expliquerait par le fait que les entreprises ont des caractéristiques non observables différentes (Rosenbaum, 2002). Il est question alors de faire varier le paramètre $\Gamma$ et de déterminer le seuil à partir duquel une modification des valeurs de $\Gamma$ pourrait remettre en cause les résultats issus des différents estimateurs. En pratique, les valeurs inférieures à 2 indiquent une sensibilité des résultats aux caractères inobservables (Clement, 2011). Dans le cadre de cet article, la variation $\Gamma$ de 1 à 2 se fera à chaque fois par tranches de 0,05 .

\subsection{Description des variables du modèle}

Les variables de résultat : La Performance de l'entreprise : Le chiffre d'affaire, le résultat net, la taille de l'entreprise (le nombre d'employés). La variable de traitement : L'accès ou non au microcrédit. Les variables de l'appariement : Les caractéristiques observées retenues pour la construction du contrefactuel des entreprises informelles bénéficiaires : Au niveau de l'entrepreneur : l'âge, le niveau d'éducation, la situation matrimoniale, le sexe. Au niveau l'entreprise : Le fond de roulement (le capital initial), le secteur d'activité.

\section{Presentation des données de l'étude}

Les données utilisées dans cette étude sont issues de l'étude portant sur «Microfinance et le Bien-être des ménages en Côte d'Ivoire (EMBEM)» avec l'appui financier et technique du Programme d'Appui Stratégique à la Recherche Scientifique (PASRES) et du projet CAPEC/CRDI. Cette étude vise à évaluer la nature, l'étendue et la direction de l'impact socio-économique des programmes de microfinance sur les emprunteurs. Le questionnaire comporte deux grandes sections : La première section est relative aux 
caractéristiques socio-économiques et démographiques des ménages, aux dépenses des ménages et à l'accès au crédit. La deuxième section est relative aux activités des entreprises informelles. L'enquête s'est déroulée du 22 Juillet au 22 Septembre 2014 et du 20 décembre 2014 au 20 Janvier 2015 dans le District autonome d'Abidjan sur un échantillon de 1213 ménages comprenant 451 bénéficiaires de crédit et 762 non-bénéficiaires et sur un sous-échantillon de 619 entreprises informelles comprenant 220 bénéficiaires et 399 non-bénéficiaires. La méthode d'échantillonnage utilisée pour la sélection du groupe cible et de contrôle est celle de l'échantillonnage à deux dégrée utilisé par le CGAP (2003). Les IMF exerçant leurs activités dans tous quartiers du district d'Abidjan, notre zone d'étude a été divisée en 13 strates, chaque quartier étant considéré comme une strate. Avant de sélectionner les strates, une liste du nombre de clients éligibles dans chaque strate (ou zone d'activité d'une IMF) c'est-à-dire les clients avec au moins 1 an et au plus 4 ans d'ancienneté dans l'IMF et ayant également introduit une demande de prêt en 2012 a été dressée. Après cette étape un échantillonnage à deux degrés a été fait pour la sélection du groupe cible avec dans un premier temps la sélection de 8 strates géographiques. Ces strates ont été sélectionnées de manière raisonnée, au lieu d'être choisie au hasard, afin de contrôler les disparités sociales et économiques qui pourrait exister dans le district entre les différents quartiers, et veiller à ce que les quartiers sélectionnés représentent la population maximale et diversifiée sur l'ensemble du district, dans un deuxième temps une sélection aléatoire a été opérée dans chaque strate pour sélectionner un groupe de ménages clients. À l'issu de ce tirage, nous obtenons un échantillon de 451 bénéficiaires et de 123 non-bénéficiaires.

Pour la sélection du groupe de contrôle deux (2) zones de dénombrement (ZD) ont été choisies de façon aléatoire dans chaque quartier sélectionné sur la base du Recensement General de la Population et de L'Habitat (RGPH) 2014 afin de procéder au dénombrement des ménages non-client. Nous faisons hypothèse que l'appartenance des deux ZD à la même localité rend les unités d'observations relativement proches du point de vue de leurs caractéristiques. Ce dénombrement a permis de constituer une liste de ménages, qui représentera la base de sondage pour la sélection des ménages non-client dans chaque quartier. La taille de l'échantillon final des ménages non-clients est de 639 proportionnellement au nombre de ménages non-clients dénombré dans chaque ZD. À l'issu de l'enquête, un échantillon de 451 ménages bénéficiaires (groupe des traités) et un échantillon de 762 ménages non-bénéficiaires (groupe de contrôle) a été constitué.

L'échantillon des entreprises a été constituer à partir des informations sur l'activité du chef de ménage. La sélection de l'entreprise s'est faite lorsque le chef de ménage déclare avoir une activité libérale informelle (ne disposant pas de déclaration fiscale) comme activité principale. Pour les bénéficiaires, la sélection des entreprises a tenu également compte de l'objet du prêt (prêt obtenu pour l'entreprise), ce qui a permis d'obtenir un échantillon de 619 entreprises informelles dont 220 bénéficiaires de microcrédit et 399 non bénéficiaires (tableau 1).

Tableau1 : Répartition de l'échantillon des entreprises informelles dans les communes sélectionnées

\begin{tabular}{|l|l|l|l|l|}
\hline \multirow{2}{*}{ Strates/communes } & Bénéficiaire & Non-bénéficiaire & Total \\
\cline { 2 - 4 } & Client & Client & Non-Client & \\
\hline Abobo & 28 & 16 & 37 & 81 \\
\hline Adjamé & 20 & 11 & 29 & 60 \\
\hline Anyama & 24 & 6 & 25 & 55 \\
\hline Attecoubé & 21 & 9 & 32 & 62 \\
\hline Bingerville & 29 & 21 & 29 & 79 \\
\hline Koumassi & 28 & 14 & 33 & 75 \\
\hline Port-Bouet & 39 & 27 & 57 & 123 \\
\hline Yopougon & 31 & 17 & 36 & 84 \\
\hline S/Total & 220 & 121 & 278 & 619 \\
\hline Total & 220 & 399 & & 619 \\
\hline
\end{tabular}

Source : L'auteur à partir des données EMBEM (2014)

\subsection{Profil des entrepreneurs de l'échantillon}

\section{Resultats et Discussions}

Les entrepreneurs bénéficiaires de crédits sont en majorité des femmes $(61,36 \%)$ contre 38,64\% d'hommes bénéficiaires (figure 2). Les hommes sont plus nombreux chez les non-bénéficiaires et représentent $77,94 \%$ de l'échantillon contre $22,06 \%$ de femmes. Cette répartition pourrait s'expliquer par le fait que la promotion de l'entrepreneuriat des femmes est au cœur des politiques mises en place par l'État de Côte d'Ivoire à travers le programme FAFCI. 


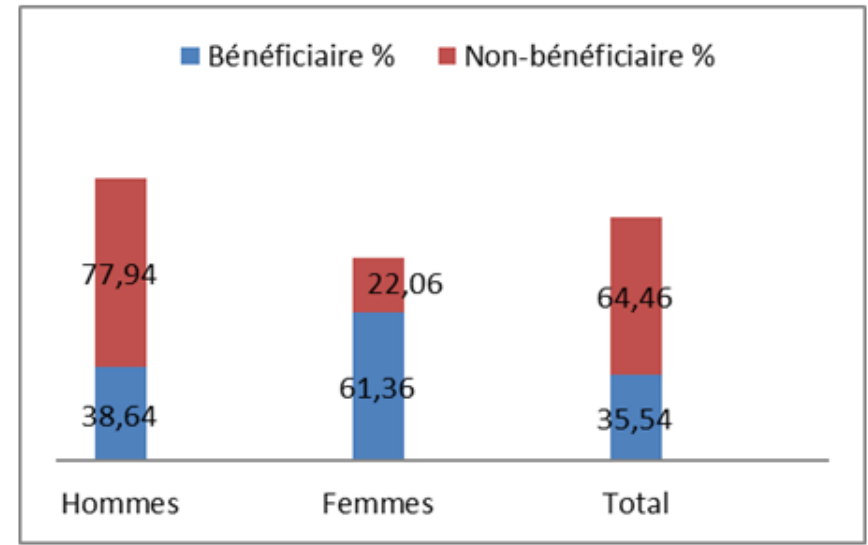

Figure (2) : Répartition des entrepreneurs selon le sexe Source: L'auteur à partir de l'EMBEM (2014)

Les entrepreneurs bénéficiaires de microcrédit sont en majorité mariés soit $56,82 \%$ de l'échantillon, cependant ce taux est dessous de ceux des non-bénéficiaires qui représentent près $66 \%$ de l'échantillon des nonbénéficiaires figure (3). Les entrepreneurs bénéficiaires célibataires sont plus nombreux soit environ $43 \%$ de l'échantillon des bénéficiaires contre $34 \%$ des micro-entrepreneurs non bénéficiaires célibataires. Près de $80 \%$ de micro-entrepreneurs de l'échantillon sont instruits, la proportion des entrepreneurs n'ayant aucun niveau est moins de $20 \%$ de l'échantillon total. Les entrepreneurs non-bénéficiaires ont un niveau d'instruction plus élevé que les bénéficiaires de crédit. En effet, 33,58 \% et 22,81\% ont respectivement le niveau secondaire et le niveau supérieur contre $33,18 \%$ et $19,50 \%$ chez les bénéficiaires. Le financement accordé aux microentreprises est en majorité dans la branche du commerce soit $51 \%$ du financement accordé par les IMF. $29 \%$ des financements accordés sont dans la branche Industrie et $20 \%$ dans la branche des services (figure 4)
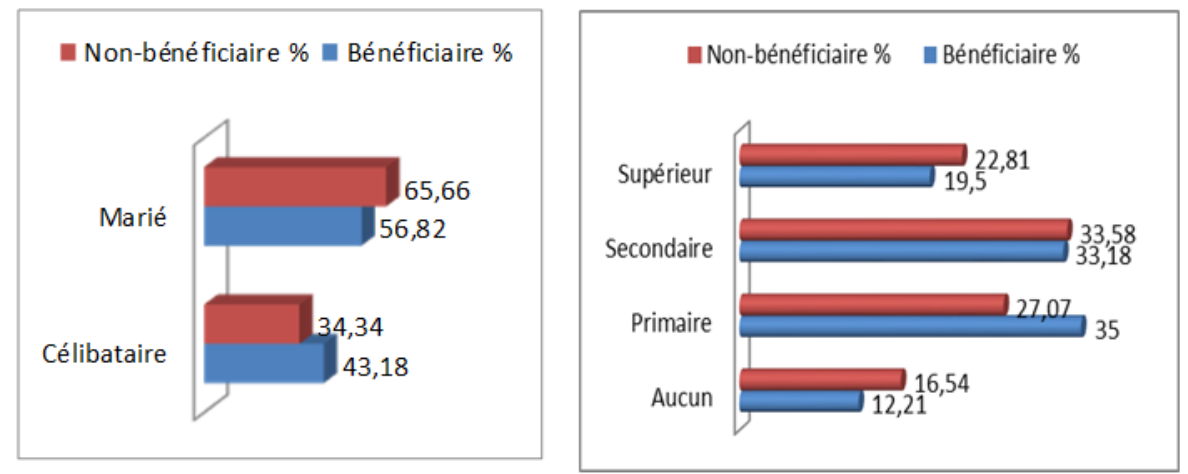

Figure (3) : Répartition des entrepreneurs selon la situation matrimoniale et le niveau d'instruction Source : L'auteur à partir des données EMBEM (2014)

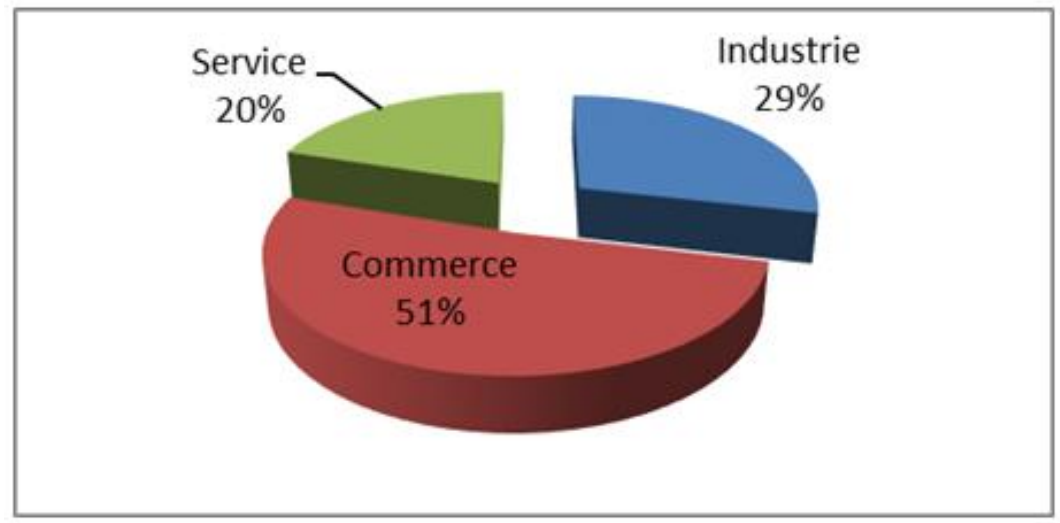

Figure (4) : Répartition des financements accordés selon les branches d'activités Source : L'auteur à partir des données EMBEM (2014) 


\subsection{Analyse de l'évolution des activités des entreprises informelles.}

Avant de présenter les résultats de l'évaluation d'impact, nous présentons la perception des entrepreneurs sur l'évolution de leurs activités et leur niveau de satisfaction relative processus d'octroi de crédit. Cette analyse se fera sur la base des trois indicateurs de performance retenus dans l'étude à savoir le chiffre d'affaire annuel, le résultat net et le nombre d'employés.

\section{- Évolution du chiffre d'affaires}

Le chiffre d'affaires est un indicateur de mesure de performance de l'activité économique d'une entreprise. La variation du chiffre d'affaires d'une entreprise est un indicateur analytique intéressant. Le tableau présente l'impact perçu par les entrepreneurs sur l'évolution de leur chiffre d'affaires après l'obtention du crédit comparativement aux entreprises non-bénéficiaires sur la même période c'est-à-dire 2 ans avant l'obtention du crédit. Les résultats permettent de constater que $68,64 \%$ des entreprises bénéficiaires ont vu leur chiffre d'affaires évoluer positivement contre 40,35\% chez non-bénéficiaires. 29,55 de micro-entrepreneurs bénéficiaires ont constaté une baisse de leur chiffre d'affaires contre $45,11 \%$ de microentreprises nonbénéficiaires. Seulement $1,8 \%$ des entreprises bénéficiaires déclarent que leur chiffre d'affaires n'a pas évolué contre $15 \%$ des entreprises non bénéficiaires.

Tableau 2: Évolution du chiffre d'affaire des bénéficiaires et des non bénéficiaire de crédit

\begin{tabular}{|l|l|l|l|}
\hline Évolution du chiffre d'affaires & $\begin{array}{l}\text { Bénéficiaire } \\
(\%)\end{array}$ & $\begin{array}{l}\text { Non-bénéficiaire } \\
(\%)\end{array}$ & Total (\%) \\
\hline Chiffre d'affaires en baisse & 29,55 & 45,11 & 39,58 \\
\hline Chiffre d'affaires resté statique & 1,82 & 14,54 & 10,02 \\
\hline Chiffre d'affaires en hausse & 68,64 & 40,35 & 50,40 \\
\hline Total (\%) & $\mathbf{1 0 0}$ & $\mathbf{1 0 0}$ & $\mathbf{1 0 0}$ \\
\hline
\end{tabular}

Source : L'auteur à partir des données EMBEM (2014)

Le tableau 2 fait ressortir l'évolution comparée du chiffre d'affaires chez les deux groupes d'entrepreneurs (bénéficiaires et non-bénéficiaires). Il se dégage clairement que l'augmentation du chiffre d'affaires a été plus significative chez les entreprises ayant bénéficié de crédit. Plus de moitié des entreprises bénéficiaires ont vu leur chiffre d'affaires évoluer positivement.

\section{- Évolution du résultat net}

L'évolution du niveau du résultat net est un indicateur qui permet d'apprécier la performance économique intrinsèque d'une entreprise. Cet indicateur permet de capter le niveau de richesse de l'entreprise. À l'instar de l'évolution constatée sur le chiffre d'affaires, l'évolution du résultat net suit les mêmes proportions. En effet ; 68,64\% des unités bénéficiaires de microcrédit ont constaté une évolution positive de leur résultat contre seulement 39,60\% chez les non-bénéficiaires. Près de 29,55\% des entrepreneurs bénéficiaires ont vu leur résultat baissé contre 46,12 \% chez les non-bénéficiaires. Seulement 1,82 \% des entrepreneurs bénéficiaires n'ont pas constaté d'évolution de leur résultat net contre 14,29\% des non-bénéficiaires.

Tableau 3 : Évolution du Résultat net des bénéficiaires et des non bénéficiaires de crédit

\begin{tabular}{|l|l|l|l|}
\hline Évolution du Résultat net & $\begin{array}{l}\text { Bénéficiaire } \\
(\%)\end{array}$ & $\begin{array}{l}\text { Non-bénéficiaire } \\
(\%)\end{array}$ & Total (\%) \\
\hline Résultat net en baisse & 29,55 & 46,12 & 40,23 \\
\hline Résultat net resté statique & 1,82 & 14,29 & 9,85 \\
\hline Résultat net en hausse & 68,64 & 39,60 & 49,92 \\
\hline Total (\%) & $\mathbf{1 0 0}$ & $\mathbf{1 0 0}$ & $\mathbf{1 0 0}$ \\
\hline
\end{tabular}

Source : L'auteur à partir des données EMBEM (2014)

L'évolution du niveau de résultat net chez les entreprises bénéficiaires est aussi restée en corrélation directe avec l'évolution du niveau du chiffre d'affaires. Plus de la moitié des bénéficiaires de microcrédit ont perçu un impact positif du crédit octroyé contre seulement $29 \%$ qui ont constaté une évolution contraire. Le microcrédit est un élément important dans la création de richesses d'une entreprise informelle.

\section{- Évolution de la taille de l'entreprise (la main d'œuvre)}

Cette section analyse l'évolution de la taille de l'entreprise captée par l'évolution de la main d'œuvre. En effet, pour augmenter sa production une entreprise doit augmenter son effectif. Il ressort de l'analyse du tableau 4 une perception positive de l'accès au microcrédit sur l'évolution de l'effectif des entreprises des bénéficiaires. En effet, 67,73\% des bénéficiaires disent avoir augmenté leur effectif après avoir eu accès au crédit contre $39,10 \%$ pour les non-bénéficiaires sur la même période. Près de $16 \%$ bénéficiaires disent n'avoir pas employé de nouvelles personnes contre $34 \%$ chez les non-bénéficiaires. Pour ce qui est de la diminution de l'effectif, près de $16 \%$ des entrepreneurs bénéficiaires déclarent avoir réduit leur effectif contre $27,32 \%$ chez les non bénéficiaires 
Tableau 4 : Évolution de la taille des entreprises bénéficiaires et non bénéficiaires.

\begin{tabular}{|l|l|l|l|}
\hline Évolution du nombre d'employés & Bénéficiaire \% & $\begin{array}{l}\text { Non-bénéficiaire } \\
\%\end{array}$ & Total \% \\
\hline Nombre d'employés en baisse & 15,91 & 27,32 & 23,26 \\
\hline Nombre d'employés resté statique & 16,36 & $\mathbf{3 3 , 5 8}$ & $\mathbf{2 7 , 4 6}$ \\
\hline Nombre d'employés en hausse & 67,73 & 39,10 & 49,27 \\
\hline Total & $\mathbf{3 5 , 5 4}$ & $\mathbf{6 4 , 4 6}$ & $\mathbf{1 0 0}$ \\
\hline
\end{tabular}

Source : L'auteur à partir des données EMBEM (2014)

Cette analyse de la perception révèle que le microcrédit a un impact positif sur la performance des activités chez près de $70 \%$ des entreprises. Après l'analyse de l'impact perçu par les bénéficiaires il importe de faire une autre analyse en se basant sur les valeurs déclarées par les entrepreneurs sur le chiffre d'affaires, le résultat net et le nombre d'employés avant et après la mise en place du crédit. Le tableau suivant présente les résultats des tests de différence de moyenne pour les trois indicateurs de performance retenue.

Tableau 5 : Différence de moyenne du niveau de performance des entreprises

\begin{tabular}{|l|l|l|}
\hline & $\begin{array}{c}2012 \text { (Avant la mise en } \\
\text { place du crédit) }\end{array}$ & $\begin{array}{l}2014 \text { (après la mise en place du } \\
\text { crédit) }\end{array}$ \\
\hline Chiffre d'affaires & $137728.1^{*}$ & $313845.2 * * *$ \\
\hline Résultats Nets & 251565.5 & $428935.8 * *$ \\
\hline Taille entreprise & 0.4255867 & $0.4275689^{* * *}$ \\
\hline
\end{tabular}

Source : L'auteur à partir des données EMBEM (2014)

$* * * \mathrm{P}<0,01, * * \mathrm{p}<0,05, * \mathrm{p}<0,1$

Le test de différence de moyenne montre qu'avant la mise en place du crédit il n'y avait pas de différence significative de performance entre les entreprises des bénéficiaires et des non-bénéficiaires. Après la mise en place du crédit, on constate une nette évolution du niveau de performance des entreprises bénéficiaires comparativement aux non-bénéficiaires.

\subsection{Résultats de l'analyse économétrique}

\subsubsection{Résultat de l'appariement sur score de propension}

Les résultats de régressions logistiques montrent que les probabilités d'obtenir du microcrédit est fonction de l'ensemble des caractéristiques de l'entrepreneur et de son entreprise telle que : le sexe, l'âge, le niveau d'instruction, le capital initial. La plupart des variables introduites dans le modèle exercent une influence statistiquement significative sur la probabilité d'appartenir ou non au groupe de traité à l'exception de la situation matrimoniale et de la branche d'activité.Pour nous assurer que pour chaque entreprise bénéficiaire, nous pouvons trouver au moins une entreprise non bénéficiaire qui ait au moins les mêmes caractéristiques, nous construisons la région du support commun des scores de propension. Afin de réaliser ceci, nous comparons les maxima et les minima de la distribution des scores de propension pour les deux groupes. Les entreprises du groupe de contrôle dont les scores de propension sont soit plus faibles, soit plus importants par rapport au minimum et au maximum des microentreprises du groupe des traités sont supprimées. La région du support commun obtenue est l'intervalle [0.12222583, 0.8983656].

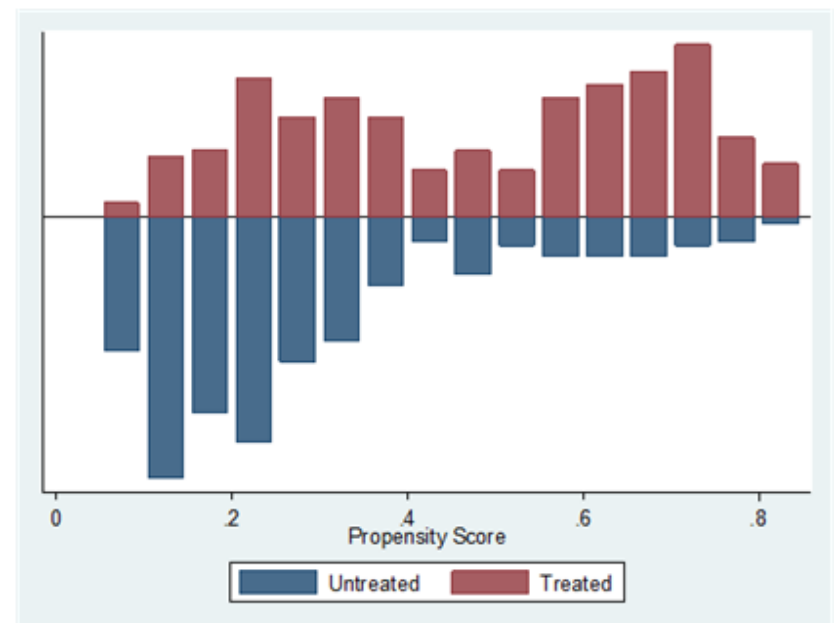

Figure 5 : Distribution des scores de propension dans la région du support commun

Source : L'auteur à partir des données EMBEM (2014) 
Après les résultats des scores de propension, l'échantillon est divisé en des blocs. Le nombre de blocs identifié est de 5. À l'intérieur de chaque bloc, l'égalité des moyennes des scores de propension des microentreprises bénéficiaires et des non-bénéficiaires est testée sous l'hypothèse d'égalité des variances. Les résultats montrent que les moyennes des scores de propension ne sont pas différentes entre les deux groupes. Le test de la propriété d'équilibre des scores de propension révèle que cette propriété est satisfaite. Le tableau (6) donne la borne inférieure, le nombre de traités et le nombre d'individus du groupe de contrôle de chaque bloc. Cette étape consiste à tester à l'intérieur de chaque bloc que les moyennes de chaque caractéristique ne diffèrent pas entre les microentreprises traitées et les microentreprises du groupe de contrôle. Si les moyennes d'une ou de plusieurs caractéristiques diffèrent dans un bloc donné, la propriété d'équilibre n'est pas satisfaite dans ce bloc. Alors celui-ci doit-être divisé en des blocs plus fins puis l'on refait le test. Si elle n'est pas encore satisfaite, l'on doit revoir la spécification du modèle en introduisant des termes d'interaction ou d'ordre supérieur. Le test d'équilibre étant vérifié, l'on peut estimer l'impact du crédit sur la performance des entreprises informelles par les méthodes d'appariement.

Tableau 6 : Nombre optimal de blocs et répartition des entreprises appariés

\begin{tabular}{|l|l|l|l|}
\hline Borne inférieure & \multicolumn{1}{|c|}{ BÉNÉFICIAIRE } & Total \\
\hline & Non & Oui & \\
\hline 0.122 & 139 & 30 & 169 \\
\hline 0.2 & 122 & 48 & 172 \\
\hline 0.4 & 61 & 70 & 129 \\
\hline 0.6 & 29 & 62 & 91 \\
\hline 0.8 & 3 & 10 & 13 \\
\hline Total & 354 & 220 & 574 \\
\hline
\end{tabular}

Source : L'auteur à partir des données EMBEM (2014)

\subsubsection{Résultats de l'évaluation de l'impact du microcrédit}

Pour déterminer l'effet moyen de l'accès au microcrédit sur la performance des entreprises, la méthode d'appariement permet de construire pour chaque entreprise bénéficiaire un contrefactuel. La probabilité d'observer deux entreprises avec exactement les mêmes valeurs du score de propension est en principe nulle puisque la distribution des scores de propension est continue. Alors un seul estimateur par appariement exact sur le score de propension est insuffisant pour évaluer l'effet moyen de l'accès au microcrédit sur la performance des entreprises informelles. Un certain nombre des estimateurs par appariement ont été suggérés dans la littérature pour contraster les résultats des individus traités avec les résultats des individus dans le groupe de comparaison (les bénéficiaires et les non-bénéficiaires). Becker et Ichino (2002) ou Caliendo et Kopeinig (2008) Dans le cas de cette étude, trois estimateurs de PSM vont être utilisé, à savoir, la méthode du plus proche voisin, la méthode de stratification et la méthode du kernel matching. Utiliser ces trois estimateurs permet d'éviter toutes les imperfections qui peuvent résulter d'une méthode simple, et d'aider également à vérifier la robustesse de l'impact estimé. Les estimateurs par appariement diffèrent par la façon de sélectionner les microentreprises du groupe de contrôle à apparier avec les microentreprises traitées et par les poids attribués aux groupes de contrôle sélectionné lors de l'estimation du résultat contrefactuel des microentreprises du groupe des traités. Toutefois, les estimateurs de l'effet moyen du traitement obtenus sur les traités sont tous consistants sous l'hypothèse d'indépendance conditionnelle (Tommaso, 2006). Le tableau (7) présente les résultats de l'estimation de l'effet moyen de traitement par trois différentes méthodes d'appariement (la méthode du plus proche voisin, la méthode de la stratification et le Kernel matching)

Tableau 7 : Résultat de l'Effet de traitement moyen-sur-traitées (ATT)

\begin{tabular}{|l|l|l|l|l|l|l|}
\hline \multirow{2}{*}{ Variables } & \multicolumn{2}{|c|}{ Proche voisin } & \multicolumn{2}{|l|}{ Stratification } & \multicolumn{2}{l|}{ Kernel matching } \\
\cline { 2 - 7 } & $A T T$ & $t$-stat & $A T T$ & $t$-stat & ATT & $t$-stat \\
\hline Chiffre d'Affaires & 0.727 & $5.048 * * *$ & 0.845 & $8.940 * * *$ & 0,842 & $7,932 * * *$ \\
\hline Résultat Net & 0.341 & $2.254 * *$ & 0.351 & $3.555^{* *}$ & 0.381 & $3.702 * *$ \\
\hline Taille entreprise & 0.397 & $2.199 * *$ & 0.441 & $2.462 * *$ & 0.412 & $2.826^{*}$ \\
\hline
\end{tabular}

Source : L'auteur à partir des données EMBEM (2014)

$* * * \mathrm{P}<0,01, * * \mathrm{p}<0,05, * \mathrm{p}<0,1$

Les résultats montrent que, quelle que soit la méthode d'estimation utilisée, le microcrédit a contribué à l'amélioration du niveau de performance des entreprises bénéficiaires puis que l'estimateur de l'effet moyen sur les différents indicateurs de performance est positif et significatif. Avec la méthode du plus proche voisin, toutes les entreprises bénéficiaires ont été appariées à 141 entreprises du groupe de contrôle, l'estimateur de l'effet moyen montre qu'il y a une augmentation (un gain) de $74 \%$ du chiffre d'affaires (au seuil de $1 \%$ ), de $61 \%$ du résultat net (au seuil de $1 \%$ ) et de 36,8\% de la taille de l'entreprise (au seuil de $1 \%$ ). Avec la méthode de stratification et Kernel matching toutes les entreprises bénéficiaires ont été appariées à 354 entreprises du 
groupe de contrôle, l'estimateur de l'effet moyen ici montre une augmentation respectivement de $83 \%$ et de $84 \%$ en moyenne du chiffre d'affaires, de $48,8 \%$ et de $52 \%$ en moyenne du résultat net et de $42 \%$ et $41 \%$ en moyenne de la taille de l'entreprise.

\subsubsection{Test de sensibilité des résultats à l'hétérogénéité inobservée.}

Dans la méthode de l'appariement sur le score de propension, il est souvent difficile de prendre en compte toutes les caractéristiques des entreprises pouvant déterminer leur probabilité à avoir accès au microcrédit. Ce qui peut entrainer un biais dû aux caractéristiques inobservées influençant l'accès au crédit pour entreprise. Ce cas de figure peut par conséquent violer l'hypothèse d'indépendance conditionnelle aux caractéristiques observables. Il est alors important de tester nos différents résultats à l'échec de cette hypothèse. Ce test s'inspire de l'analyse de la sensibilité développée par Rosenbaum (2002). Les résultats sont présentés dans le tableau 8 ci-dessous pour les différents estimateurs significatifs obtenus. Les résultats de l'estimation de l'impact de l'accès au microcrédit (attnd $=72 \%$; atts $=84 \%$; attk $=84 \%$ ) sur le chiffre d'affaires des entreprises informelles et sur le résultat net (attnd $=34 \%$; atts=35\%; attk =38\%) sont robuste à l'échec de l'hypothèse d'indépendance conditionnelle aux caractéristiques observables. En effet, le seuil à partir duquel $\Gamma$ (gamma) n'est pas significatif (>10\%) est inférieur respectivement à 1,95 et 1,45 (le point à partir duquel l'effet du traitement peut être remis en cause). Autrement dit, il faudrait un changement de $95 \%$ et de $45 \%$ dans le rapport de chance d'avoir accès au microcrédit pour biaiser l'ATT à cause des caractéristiques non observables. Par contre, l'impact sur la taille de l'entreprise (attnd $=39 \%$; atts $=44 \%$ et attk $=41 \%$ ) n'est pas robuste à l'hétérogénéité. En effet, le seuil de gamma à partir duquel l'ATT peut être contesté est de 1,30. Autrement dit, il faudra un changement de plus $30 \%$ pour biaiser l'ATT sur la taille de l'entreprise. Ce résultat est donc à prendre avec précaution. Toutefois, le test de sensibilité ne rend pas compte de l'existence ou non d'un biais dû aux caractéristiques non-observables des entreprises, il permet simplement d'apprécier la variation des estimations dans le cas où il existerait un biais sur les inobservables.

Tableau 8 : Résultat du Test de Rosenbaum

\begin{tabular}{|l|l|l|l|}
\hline$\Gamma$ & Chiffre d'affaires & Résultat Net & Taille entreprise \\
\hline 1 & $1.4 \mathrm{e}-07$ & .000259 & .004309 \\
\hline 1,05 & $6.7 \mathrm{e}-07$ & .000754 & .009131 \\
\hline 1,1 & $2.6 \mathrm{e}-06$ & .001918 & .017543 \\
\hline 1,15 & $9.1 \mathrm{e}-06$ & .004354 & .030967 \\
\hline 1,20 & 0.000027 & .008944 & .050785 \\
\hline 1,25 & 0.000074 & .016836 & $\mathbf{. 0 7 8 1 1 4}$ \\
\hline 1,30 & .000183 & .02935 & .113596 \\
\hline 1,35 & .000415 & .047816 & .157269 \\
\hline 1,40 & .000868 & $\mathbf{. 0 7 3 3 7 8}$ & .208524 \\
\hline 1,45 & .001697 & .106791 & .266173 \\
\hline 1,50 & .003121 & .148275 & .328582 \\
\hline 1,55 & .00543 & .197445 & .393857 \\
\hline 1,60 & .008986 & .253326 & .460037 \\
\hline 1,65 & .014214 & .314462 & .525266 \\
\hline 1,70 & .021578 & .379068 & .587928 \\
\hline 1,75 & .031558 & .445212 & .646728 \\
\hline 1,80 & .044607 & .510999 & .700735 \\
\hline 1,85 & .061124 & .574714 & .749374 \\
\hline 1,90 & $\mathbf{. 0 8 1 4 0 9}$ & .634933 & .792392 \\
\hline 1,95 & .105645 & .690582 & .829813 \\
\hline 2 & .133868 & .740948 & .861867 \\
\hline
\end{tabular}

Source : Calcul de l'auteur à partir des données EMBEM (2014)

L'impact positif du chiffre d'affaires chez les entreprises bénéficiaires de microcrédit peut s'expliquer par l'économie d'échelle et l'économie réalisée sur les prix en raison de la fluctuation des coûts des matières premières et/ou des marchandises. En effet, le crédit a permis aux entrepreneurs bénéficiaires d'acheter des marchandises et/ou de la matière première en grande quantité, ce qui a fait baisser le prix unitaire en leur faveur. L'octroi du crédit a certainement permis d'acheter les matières premières et marchandises au moment où les prix sont les plus abordables. Cela leur a permis d'augmenter la production ou les stocks de marchandises. L'économie réalisée sur les achats a permis de baisser les prix de vente. L'augmentation de l'offre et la baisse du prix de cette offre ont eu pour conséquence directe l'augmentation des chiffres d'affaires. Ces résultats sont en conformité avec ceux des travaux de Gubert et al (2004); Koloma et Ratsimalahelo (2015). L'augmentation du chiffre d'affaires a un impact positif sur le niveau du résultat net qui mesure l'enrichissement de l'entreprise. Par conséquent, à partir de l'évolution du chiffre d'affaires, nous pouvons dire que les entrepreneurs bénéficiaires de crédit ont eu plus d'opportunités de s'enrichir. L'impact positif du microcrédit sur le résultat net montre que les entreprises bénéficiaires se sont majoritairement plus enrichies que les entreprises témoins. Ce 
qui témoigne de la contribution du crédit comme élément favorisant l'enrichissement des entreprises. Cependant cette augmentation du résultat net des entreprises bénéficiaires n'a pas permis une forte croissance de la main d'œuvre. En effet, les bénéfices réalisés par les entreprises ne sont pas suffisants pour accroitre la main-d'œuvre au sein de ces entreprises. Ces résultats confirment ceux de Sebstad et Chen (1996).

\section{Conclusion Et Recommandation}

Cette étude a évalué le lien entre l'accès au microcrédit et la performance des entreprises informelles en Côte d'Ivoire en utilisant la méthode quasi-expérimentale basée sur la méthode d'appariement sur score de propension. Les résultats des différents estimateurs révèlent un impact positif et significatif du microcrédit sur la performance des microentreprises informelles. La performance des entreprises informelles bénéficiaires semble en effet avoir fortement évolué avec le recours au crédit, en termes de chiffre d'affaires avec un gain de $80 \%$ en moyenne. Cependant cette évolution est moins forte au niveau du résultat net et du nombre d'employé avec un gain respectivement d'environ $35 \%$ et $40 \%$ en moyenne. Le microcrédit a certes permis aux bénéficiaires d'améliorer le fonds de roulement pour les uns et le matériel de production pour les autres. Cela leur a permis d'augmenter leur capacité de production et de vente. Les marges ainsi réalisées ont contribué à enrichir les entreprises bénéficiaires contrairement à ceux qui n’ont pas bénéficié de crédit. Du point de vue des bénéficiaires, le microcrédit a un impact significatif et positif sur leurs entreprises pour environ $70 \%$ des entrepreneurs. Cependant environ $29 \%$ des entrepreneurs disent avoir vu le niveau de performance baissé après l'accès au microcrédit et seulement $1 \%$ affirment ne percevoir aucun impact sur leurs activités. Un crédit qui ne profite pas au bénéficiaire est un crédit qui a été mal géré (orienté vers une autre destination ou inopportune) ou affecté par un phénomène extérieur (maladie du promoteur, catastrophe naturelle, vol, incendie...) ou affecté au remboursement du crédit. En effet, pour $30 \%$ des bénéficiaires la politique de remboursement du crédit constitue l'une des premières causes d'insatisfaction. Ceux-ci considèrent la politique de remboursement comme une contrainte dans le développement de leurs activités bien que le chiffre d'affaires connaisse une hausse considérable. Ces résultats obtenus permettent de formuler quelques recommandations à l'endroit :

Des responsables de structures de Microfinance :

- $\quad$ Mise en place d'un cadre d'échange et de suivi des activités financées en vue d'être informé des difficultés que rencontrent les entrepreneurs dans la conduite de leurs activités. Ce qui devrait permettre de revoir la politique de remboursement de crédit.

- $\quad$ Mise en place d'un programme de formation qui renforce les capacités managériales des entrepreneurs (et non pas seulement leur capacité financière). Cette mesure devrait permettre aux entrepreneurs d'être des entrepreneurs dynamiques qui à terme pourraient intégrer le secteur formel de l'économie.

Des autorités :

- $\quad$ Mise en place de plusieurs fonds à l'endroit des personnes ayant une activité génératrice de revenus ou voulant créer une activité en renforçant les programmes qui existent déjà tel le FAFCI (Fonds d'Appui aux Femmes de Côte d'Ivoire). En effet, cette étude sur l'évaluation d'impact de l'accès au crédit a permis de voir la contribution positive du microcrédit sur l'évolution des activités du secteur informel.

- $\quad$ Mise en place d'un cadre de concertation État secteur informel qui permettra à l'État de Côte d'Ivoire d'évaluer les difficultés de ce secteur.

L'évaluation d'impact menée dans le cadre de cette étude reste partielle, il convient pour rendre nos résultats plus robustes de procéder à un autre passage auprès du même échantillon en vue de faire une analyse dynamique de l'impact du microcrédit.

References

[1] A, Aakvik. Bounding a matching estimator: The case of a Norwegian training program. Oxford Bulletin of Economics and Statistics, vol. 63, 2001, 115-143.

[2] A. A. Mbaye, Le rôle du secteur informel pour la croissance, l'emploi et le développement Durable, document de travail OIF, 2014

[3] A. K. Ghalib, I.Malki, K.S.Imai, Microfinance and its role in household poverty reduction : findings from Pakistan, Brooks World Poverty Institute, 2012, working paper 173

[4] A.Lecocq, M.Ammi et E. Bellarbre, Le score de propension : un guide méthodologique pour les recherches expérimentales et quasi expérimentales en éducation, Mesure et évaluation en éducation, vol. 37, $\mathrm{n}^{\circ} 2,2014$, p. 69-100.

[5] AFD, Amartya Sen : Un économiste du développement? (Agence Française de Développement Département de la Recherche. 2008)

[6] AFD, Rapport annuel 2012 de l'Agence Française de Développement, 2012

[7] AGEPE (2012), rapport de l'Enquête sur l'emploi en Côte d'Ivoire, AGEPE 2012

[8] B.Augsburg, , R. D. Haas, Y. Harmgart, and C. Meghir. Microfinance, poverty and education. IFS working paper. 2012

[9] Banerjee, V.Abhijit, E. Duflo, R.Glennerster, and C.Kinnan. 2015. The Miracle of Microfinance? Evidence from a Randomized Evaluation. American Economic Journal : Applied Economics, 7 (1): 2015, p.22-53.

[10] B. Crépon, , E. Duflo, F. Devoto, and Y. Pariente, Impact of microcredit in rural areas of Morocco: Evidence from a randomized evaluation. $J-P A L$ working paper2011

[11] B.E Coleman., Microfinance in Northeast Thailand: Who Benefits and How Much? , Economic and Research Department Paper Series n 9, Asian Development Bank, 2002 35p 
[12] B.Gutiérrez-N , C. Serrano, C.M. Molinero , Microfinance Institutions and Efficiency, Omega International Journal of Management Science. 2005

[13] B.MkNelly and C.Dunford Impact of Credit with Education on Mothers and Their Young Children's Nutrition: CRECER Credit with Education Program in Bolivia , Freedom from Hunger Research Paper No. 5 (Davis, Calif.: Freedom from Hunger, 1999)

[14] C.Barnes, G.Gaile et R. Kimbombo, Impact of Three Microfinance Programs in Uganda, USAID-AIMS paper, Management of Systems International, 2001

[15] CGAP, Outil de l'évaluation de la pauvreté en Microfinance, Série « Outil technique » n 5, Mars 2003

[16] D. Fougère, Les méthodes micro-économétriques d'évaluation, Banque de France, NER - 2007, E \# 166

[17] D.S. Karlan and J. Zinman, Microcredit in Theory and Practice: Using Randomized Credit Scoring for Impact Evaluation, SCIENCE VOL 332, 2011 www.sciencemag.org

[18] D.S. Karlan, Microfinance Impact Assessments: The perils of Using New members as a Control Group , mimeo, AIMS ,2001

[19] Direction de la Microfinance (DM), Rapport annuel de la Commission Nationale de la Microfinance Côte d'Ivoire, Direction Générale du Trésor et de la Finance Publique ,2012

[20] ENSETE, Rapport de l'Enquête Nationale sur la Situation de l'Emploi et du Travail des Enfants en Côte d'Ivoire, Agence emploi jeune/ INS , 2013

[21] F.Gubert et F.Roubaud (2004), Le financement des très petites entreprises urbaines : étude d'impact d'un projet de microfinance à Madagascar, STATECO N98, 2004

[22] G.A. Barboza., H. Barreto, Learning by Association: Microcredit in Chiapas, Mexico, Contemporary Economic Policy, vol.24, $\mathrm{n}^{\circ} 2$. 2006

[23] H.Zaman, Assessing the Poverty and Vulnerability Impact of Micro-Credit in Bangladesh: A Case Study of BRAC, (Banque mondiale. 2000),

[24] I.GUERIN, Les effets insoupçonnés de la microfinance, Travail, genre et sociétés, $\mathrm{n}^{\circ}$ 25, 2011, pp. 61-79.

[25] J.C. Brau ; G. M. Woller, Microfinance : A Comprehensive Review of the Existing Literature, Journal of Entrepreneurial Finance and Business Ventures: Vol. 9: Iss. 1, 2004, pp. 1-28.

[26] JH.Conning. Outreach, Sustainability and Leverage in Monitored and Peer-Monitored lending., Journal of Development Economics 60: 1999, 51-77

[27] J. Ledgerwood, , Manuel de Microfinance : une perspective institutionnelle et financière, (Washington. DC, Banque Mondiale, 1998)

[28] J. Morduch et B. Haley, Analysis of the Effects of Microfinance on Poverty Reduction , NYU Wagner Working Paper No. 1014, June 28, 2002

[29] J. Morduch, The Microfinance Promise, Journal of Economic Literature, vol.37, December 1999.

[30] J.Morduch, The Microfinance schism, World Development, vol.28, Nº4, pp.617-629, 2000.

[31] J.Sebstad and G.,Chen, Overview of studies on the impact of microenterprise credit, AIMS Brief No. 1, USAID, Washington . 1996

[32] K.Wright-Revolledo, Monitoring the Diversity of the Poverty Outreach and Impact of Microfinance: A Comparison of Methods Using Data from Peru, DEVELOPMENT POLICY REVIEW, Volume 23, Issue 6, November 2005, Pages: 703-723,

[33] M.Angelucci, , D. Karlan, and J. Zinman. Win some lose some? Evidence from a randomized microcredit program placement experiment by Compartamos Banco. J-PAL working paper. 2012

[34] M. Clement. Remittances and Household Expenditure Patterns in Tajikistan: A Propensity Score Matching Analysis. Asian Development Review, Vol. 28, 2011

[35] L.Ejigu, Performance Analysis of a Sample Microfinance Institutions of Ethiopia.International, NGO Journal, 4(5), 2009, PP.287298.

[36] M Lelart, .De la finance informelle à la microfinance. Éditions des archives contemporaines, (Agence Universitaire de la Francophonie (AUF). 2006.)

[37] M Pitt.and S.Khandker, , The impact of Group-Based Credit Programs on Poor Households in Bangladesh: Does the Gender of Participants Matter?, Journal of Political Economy, vol. 106, n5, pp. 1998, 958-977.

[38] M.Caliendo, and S.Kopeinig,. Some practical guidance for the implementation of propensity score matching . Journal of Economic Surveys 22(1), 2008, 31-72.

[39] M.Labie, Microfinance : un état des lieux , Mondes en Développement, Vol. 32/2-n 126, 2004, pp. 9-23.

[40] M.Yunus ., Moingeon B. \& Lehmann-Ortega L, "Building Social Business Models: Lessons from the Grameen Experience", AprilJune, vol 43, 2010, n $^{\circ}$ 2-3, Long Range Planning, p. 308-325

[41] O. Attanasio, B. Augsburg, R. De Haas, E. Fitzsimons, and H. Harmgart, The Impacts of Microfinance:Evidence from JointLiability Lending in Mongolia, American Economic Journal: Applied Economics, 7(1): 2015, p.90-122

[42] OIT, La Microfinance pour le travail, (Bureau international du Travail - Genève: BIT, 2015)

[43] P. J Gertler., S. Martinez, P Premand, L. B. Rawlings., C. M. J. Vermeersch, L'évaluation d'impact en pratique, Banque internationale pour la reconstruction et le développement, (Banque mondiale, 2011)

[44] P. Randriamanampisoa, Microcredit et gestion des risques, une grille de lecture par les capabilites : Le cas des ménages ruraux des hautes terres de Madagascar, Thèse de Doctorat Université de Montpellier I,2011

[45] P.Rosenbaum,, Observational Studies (New York: Springer, 2nd edition, 2002)

[46] P.Rosenbaum, and D.Rubin, The Central Role of the Propensity Score in Observational Studies for Causal Effects. Biometrika, 70,1983, 41-55.

[47] R. H. Dehejia, and S.Wahba,. Propensity score-matching methods for non-experimental causal studies. The Review of Economics and Statistics 84(1), 2002, 151-161.

[48] R.Montgomerry, and J.Weiss, Great expectations: Microfinance and poverty reduction in Asia and Latin America . ADBI Research Paper Series, 63. Tokyo, Japan: ADBI Publishing, 2005.

[49] Roodman et J. Morduch, , The Impact of Microcredit on the Poor in Bangladesh: Revisiting the Evidence, Center for Global Development, Working Paper $\mathrm{n}^{\circ} 174$, June. (2009)

[50] S. O. Becker, and A.Ichino,. Estimation of average treatment effects based on propensity scores. The Stata Journal 2(4), 2002, 358377.

[51] S.Khandker, Fighting Poverty with Microcredit: Experience in Bangladesh, New York, Oxford University Press, Inc. 1998

[52] Y. Koloma et Z. Ratsimalahelo, Jeunes, accès au microcrédit et performance des microentreprises : une évidence au Mali, Centre de Recherche sur les Stratégies Economiques(Crese), working paper,2015

[53] Wooldridge, M. Jeffrey. Econometric Analysis of Cross Section and Panel Data.Cambridge, Mass.: (MIT Press. 2002) 


\section{Annexes}

1 : Résultat de l'estimation du modèle de traitement

Tableau de Résultat de l'estimation du modèle Logit

\begin{tabular}{|c|c|c|c|c|c|c|}
\hline \multirow{2}{*}{$\begin{array}{l}\text { variables } \\
\text { age }\end{array}$} & \multirow{2}{*}{$\begin{array}{l}\text { Coefficient } \\
0.0539\end{array}$} & \multirow{2}{*}{$\begin{array}{l}\begin{array}{l}\text { Écart- } \\
\text { type }\end{array} \\
0.0106\end{array}$} & \multirow{2}{*}{$\begin{array}{l}\mathrm{Z} \\
5.05\end{array}$} & \multirow{2}{*}{$\begin{array}{l}\mathrm{P}>\mid \mathrm{z} \\
0.000^{* * * *}\end{array}$} & \multicolumn{2}{|c|}{ [95\% Conf. Interval] } \\
\hline & & & & & 0.0330 & 0.0748 \\
\hline sexe & 1.676 & 0.2038 & 8.22 & $0.000^{* * *} *$ & 1.2766 & 2.0756 \\
\hline niveau_inst & 0.1797 & 0.0807 & 2.22 & $0.026 * *$ & 0.0213 & 0.3380 \\
\hline Situat matri & -0.1927 & 0.2113 & -0.91 & 0.362 & -0.6069 & 0.2214 \\
\hline lcap & 0.1758 & 0.0845 & 2.08 & $0.037 * *$ & 0.0101 & 0.3414 \\
\hline Branche_activ & -0.0980 & 0.0631 & -1.55 & 0.120 & -0.2217 & 0.0256 \\
\hline constante & -7.1264 & 1.3072 & -5.45 & $0.000^{* * *}$ & -9.6886 & -4.5643 \\
\hline observations & \multicolumn{6}{|l|}{619} \\
\hline LR chi2(7) & \multicolumn{6}{|l|}{133.70} \\
\hline Prob > chi 2 & \multicolumn{6}{|l|}{0.0000} \\
\hline Loglikelihood & \multicolumn{6}{|l|}{-335.95259} \\
\hline
\end{tabular}

Source : L'auteur à partir des données EMBEM (2014)

\section{2 : Résultats du scores de propension}

Tableau des Scores de propension estimés

\begin{tabular}{|l|l|l|}
\hline & Percentiles & smallest \\
\hline $1 \%$ & 0.1249316 & 0.1222258 \\
\hline $5 \%$ & 0.1354866 & 0.1230837 \\
\hline $10 \%$ & 0.1490299 & 0.1238745 \\
\hline $25 \%$ & 0.1893037 & 0.1248375 \\
\hline $50 \%$ & 0.3072801 & \\
\hline & & largest \\
\hline $75 \%$ & 0.5622537 & 0.8428438 \\
\hline $90 \%$ & 0.6861844 & 0.8447539 \\
\hline $95 \%$ & 0.7583902 & 0.8545187 \\
\cline { 1 - 2 } $99 \%$ & 0.8385197 & 0.8983656 \\
\hline Observations & 574 & \\
\cline { 1 - 2 } Sum of Wgt & 574 & \\
\cline { 1 - 2 } Mean & 0.3708854 & \\
\cline { 1 - 2 } Std. Dev & 0.2137015 & \\
\cline { 1 - 2 } Variance & 0.0456683 & \\
\cline { 1 - 1 } Skewness & 0.5607335 & \\
\cline { 1 - 1 } Kurtosis & 2.042464 & \\
\cline { 1 - 1 } & & \\
\hline
\end{tabular}

Source : Calcul de l'auteur 